\title{
A novel numerical scheme for the analysis of effects of surface roughness on EHL line contact with couple stress fluid as lubricant
}

\author{
BHARATI M SHETTAR ${ }^{1}$, P S HIREMATH ${ }^{1}$ and N M BUJURKE ${ }^{2, *}$ \\ ${ }^{1}$ KLE Technological University, Vidyanagar, Hubballi 580 031, India \\ ${ }^{2}$ Department of Mathematics, Karnatak University, Dharwad 580003 , India \\ e-mail: bharati_shettar@bvb.edu; pshiremath@bvb.edu; bujurke@yahoo.com
}

MS received 29 June 2016; revised 24 April 2017; accepted 7 September 2017; published online 28 June 2018

\begin{abstract}
In this article, a Jacobian-free Newton Multigrid (JFNMG) method is used for obtaining the solution of isothermal, steady and compressible elastohydrodynamic lubrication (EHL) line contact problem with surface roughness. The lubricant is a couple stress fluid. A finite difference scheme is used for the solution of EHL equations. The proposed JFNMG method, for the solution of resulting nonlinear system of algebraic equations, comprises nonlinear Newton iterations on the outer loop and linear multigrid iterations on the inner loop. It overcomes the limitations of conventional schemes for the investigation of the problems covering wide range of physical parameters of interest. For increasing values of couple stress parameter, there is an increase in minimum film thickness and considerable decrease (in height as well as spread) of pressure spike. Also, the sensitivity of height and spread of pressure spike as a function of load, couple stress parameter and other parameters are investigated.
\end{abstract}

Keywords. Elastohydrodynamic lubrication; couple stress fluid; surface roughness; Newton multigrid; inexact Newton.

\section{Introduction}

In elastohydrodynamic lubrication (EHL), application of heavy load on the bearings develops high pressure in the lubricant and deforms contacting surfaces and as such film thickness becomes a function of pressure developed. Also, the viscosity and density of the lubricant depend nonlinearly on pressure developed. The pressure developed in the film region $(0.3 \mathrm{Gpa}$ to $3.0 \mathrm{Gpa})$ and the corresponding minimum film thickness attained is in a wide range of scale (micrometer). These aspects require special skills to conduct experiments for finding pressure and film profiles. Theoretical predictions made in EHL analysis are validated based on accurate experimental data. Early developments of EHL theory and experiments presented in [1] are of interest. Latest experiments include (improvements over optical interferometer), namely, image analysis procedure, introduction of spacer layer, high speed video camera, etc. Mathematical modeling of isothermal EHL problems comprises Reynolds equation, film thickness equation and force balance equation. In addition, viscosity-pressure and density-pressure (for compressible lubricant) relations have to be considered. The boundary conditions to be satisfied are inlet condition, outlet condition and cavitation condition on pressure. In the discrete form, the coupled nonlinear algebraic equations have to

*For correspondence be solved using suitable methods. Simple Gauss-Seidel iteration method [2] is used to solve EHL equations covering a variety of problems (elliptical parameter results, fully flooded results). Some of the salient features and earlier contributions are given in the book [3]. For the line contact EHL problem, governing equations are solved later using Gauss-Seidel scheme and expression for minimum and central film thickness are derived [4], which are widely used. More refined contributions in [5] and [6], on the solution scheme are widely used. The method (Gauss-Seidel) requires under-relaxation parameter for convergence and is unstable for large load condition. For solving difference equations simultaneously, Newton-Raphson method is used [7] and sound mathematical justifications of the pressure spike [8] as well as stability of the scheme treating it as nonlinear complementary problem are given in [9] and [10]. But the scheme has drawback in dealing with Jacobian matrix, requiring huge storage which is not stable for large load conditions. Finite element methods (and recent discontinuous Galerkin methods) are more versatile which can accommodate all aspects of the EHL problems (higher order cases, transient case, etc), but it requires bigger computing system and (tedious) programming skills. However, in one of the recent articles, [11] authors alleviate these fears and stress that finite element method is equally amenable to common users on a PC. An important development in these solution schemes of EHL problem is the emergence of 
multigrid techniques [12] combined with Gauss-Seidel relaxation scheme. It accelerates the convergence of solution scheme and enables implementation of the scheme on smaller computers. Later developments [13-15], give multilevel, multi-integration for the fast evaluation of elastic deflection equation. The striking strategy here is that multigrid eliminates both high and low frequency components of errors using sequence of grid points obtained via intergrid operators. Multigrid scheme for the solution of nonlinear system of equations is Full Approximation Scheme (FAS) with full multigrid [16]. The interest in the detailed investigation of pressure-spike, its location, height and spread is not only of academic nature, but also is of practical importance as it affects surface fatigue and early failures of rolling element bearings. Detail investigation of the nature of pressure spike (its height and spread) are made [17-20] using different methods. Review articles [21, 22] present current status of EHL studies. In recent studies [23, 24], authors demonstrate the robustness of Krylov subspace methods (restart GMRES) for the analysis of EHL line contact problems. The precise nature of Petrusevich pressure spike as a function of load and speed parameters are presented.

\subsection{Couple stress fluid}

For the improved performance of EHL contacts, the lubricant plays a vital role. Addition of long chain polymers to base oil enhances the quality of lubricants. Experimental studies [25-28], have demonstrated considerable load enhancement and friction reduction in bearings by this process. Later, film forming properties of lubricant-base oil mixtures in EHL contact are given in [29, 30]. The flow behavior of Newtonian lubricant with suitable additives can be described by the simplest theory of couple stress fluid, namely, Stokes' model [31]. The Stokes model accounts for polar effects, couple stresses and body couples on the fluid. Exhaustive review of literature [32] of recent articles on couple stress fluid as lubricant in hydrodynamic as well as in EHL studies is of much use. In [33] steady state analysis of incompressible line contact EHL problem with couple stress fluid model as lubricant is discussed. Accurate empirical formula for the film thickness of EHL elliptical contacts using couple stress lubricant [34] is a good substitute to earlier ones. The influence of couple stress on steady state thermal EHL line contact problem and, later investigation of compressible EHL line contact problem to demonstrate the combined influence of couple stress lubricant with wavy surface roughness on lubrication characteristics are presented in [35, 36] respectively. For point contact problem [37] the effects of surface roughness and couple stress lubricant on EHL characteristics using average flow model equation are obtained. In one of the recent studies [32] accurate asymptotic formula for central and minimum film thickness with couple stress lubricant for finite EHL problem are given.

\subsection{Surface topology}

The surface topology of heavily loaded (EHL) mechanical components (viz., gears, cams, followers, rolling bearings) are of rough structure even with most efficient surface finish. This surface asperity affects lubrication characteristics such as pressure, film thickness and friction. The influence of roughness is investigated either with stochastic models or deterministic models. In the former, roughness is modeled through stochastic components such as flow factors which predict average pressure, average film profiles, etc., but fail to provide details of localized peak pressure, exact film shape, etc., which are important in proposing suitable design parameters of bearings. Notable earlier stochastic studies on this topic are presented in references [38-40]. To overcome the drawbacks of stochastic models, useful studies on surface roughness effects in EHL contacts using deterministic model are made [41]. Recently, Carreau type shear thinning lubricant model is used [42] to present surface roughness effect in pure sliding EHL line contact. The influential work on fatigue life theory [43] of bearings establishes relation between surface features, the associated stress and fatigue life. To overcome the limitations of stochastic models, many deterministic models that account for real surface topology (for thermal, mixed type, etc.) have been proposed and developed. Some of the important contributions on this latter category are presented in [44-46]. These models are more useful for the design, performance analysis and life improvement of bearings.

Jacobian-free Newton multigrid (JFNMG) method JFNMG method is a nested iterative scheme for the solution of nonlinear system of algebraic equations. We use Jacobian-free Newton multigrid method for solving nonlinear system of algebraic equations arising from discretization of equations modeling EHL problem. System of nonlinear equations is of the form

$$
F(x)=0
$$

where $F: R^{n} \rightarrow R^{n}$ is a nonlinear continuously differentiable function. Newton type methods are robust for the solution of equation (2.1) with suitable initial approximation. Newton method iteratively builds up a sequence of approximate solutions $\left\{\mathrm{x}^{\mathrm{k}}\right\}$ and the $(\mathrm{k}+1)$ th iterate is given by

$$
x^{k+1}=x^{k}+\delta x^{k}
$$

The update direction $\delta x^{k}$ is the solution of system of linear equations

$$
J\left(x^{k}\right) \delta x^{k}=-F\left(x^{k}\right), \quad \mathrm{k}=0,1, \ldots
$$

$J\left(x^{k}\right)$ is the Jacobian of $\mathrm{F}$ at $\mathrm{x}^{\mathrm{k}}$, with the initial approximation $\mathrm{x}^{0}$ and nonlinear iteration index $\mathrm{k}$. In favorable cases, $\left\{\mathrm{x}^{\mathrm{k}}\right\}$ converges to the solution $\mathrm{x}$ of equation (2.1) quadratically. This local convergence depends 
mostly on a good initial approximation for the solution of linear system of equations (2.3). Various methods are used for this purpose. We plan to use (linear) multigrid method. Once $\delta x^{k}$ is obtained, the Newton direction is determined, which is controlled by backtracking in not going too far (i.e., sufficiently decrease $\delta x^{k}$ to find Newton step). An inexact Newton method [47] for solving linear system of equations (2.3), up to an accuracy controlled by the forcing term $\eta_{k} \in(0,1)$, is to find $\delta x^{k}$ such that

$$
\left\|F\left(x^{k}\right)+F^{\prime}\left(x^{k}\right) \delta x^{k}\right\| \leq \eta_{k}\left\|F\left(x^{k}\right)\right\|
$$

where $F\left(x^{k}\right)+F^{\prime}\left(x^{k}\right) \delta x^{k}$ is the residual of equation (2.3) as well as local linear model of $\mathrm{F}$ evaluated at $\mathrm{x}^{\mathrm{k}}$. The forcing term $\eta_{k}$ sets the accuracy of the update direction $\delta x^{k}$, with proper selection of $\left\{\mathrm{x}^{\mathrm{k}}\right\}$. Inexact Newton method converges locally and super linearly. Further, for the inexact Newton global convergence (with arbitrary initial condition), we use Armijoline search method [47]. In EHL problems, Hertz's pressure serves as good initial approximation and as such it is easy to set the forcing term $\eta_{k}$ for backtracking Newton direction.

\subsection{Approximation of Jacobian (matrix)-vector product}

For a scalar problem (2.1), with $\mathrm{n}+1$ equations in $\mathrm{n}-1$ unknowns, we let

$$
\begin{aligned}
& F(x)=\left\{F_{1}, F_{2}, F_{3}, \ldots, F_{n}, F_{n+1}\right\} \quad \text { and } \\
& \quad x=\left\{x_{1}, x_{2}, x_{3}, \ldots, x_{n}, x_{n+1}\right\}
\end{aligned}
$$

To find the exact expression for $J$, it is a tough job in many situations but the Jacobian (matrix)-vector product (which is required in each iteration of Gauss-Seidel) can be approximated by

$$
J v \cong \frac{[F(x+\in v)-F(x)]}{\in}
$$

where $\epsilon$ is small and its optimum value is $10^{-9}$ in 64 -bit double precision [48]. Main advantage is that, without forming or storing the true/exact Jacobian, it gives vector that approximates the matrix vector multiplication (required at each iteration).

The layout of remaining part of the article is as follows. In the next section, equations representing the proposed model and the relevant relations are given. This is followed by presenting discretized forms of the equations and solution procedure. Later, the solutions and the results obtained are presented in graphs with relevant discussion and conclusions.

Governing equations and other relations The isothermal EHL line contact problem models the lubricant flows between two cylinders rotating under an applied load. The physical problem is described by the coupling of Reynolds equation, for the flow of lubricant, and elastic deformation equation of cylinders. For a steady isothermal couple stress lubricant flow, the dimensionless form of pressure $P$ is described by Reynolds equation, [36],

$$
\frac{d}{d X}\left(\in(P) \frac{d P}{d X}\right)-K \frac{d}{d X}(\rho H)=0
$$

Where $\epsilon=\frac{\rho H^{3}}{\eta \xi}, \xi=\left[1-\frac{12}{\Omega^{2}}+\frac{24}{\Omega^{3}} \tanh \left(\frac{\Omega}{2}\right)\right]^{-1}, \Omega=\frac{8 W H}{\pi L_{m}}$, $K=\frac{\left(3 U \pi^{2}\right)}{4 W^{2}}, P(X)$ and $H(X)$ are unknown pressure and film thickness, respectively, $L_{m}$ is a dimensionless couple stress parameter. The film thickness function incorporating the sinusoidal roughness term in dimensionless form is given by

$$
H(X)=H_{0}+\frac{X^{2}}{2}+\bar{v}+\bar{a} \sin \left(\frac{2 \pi X}{l}\right)
$$

where $\bar{v}=\frac{v R}{b^{2}}$ is the dimensionless surface displacement given by $\bar{v}=-\frac{1}{2 \pi} \int_{X_{1}}^{X_{c}} P \ln (X-S)^{2} d s, \frac{X^{2}}{2}$ defines the undeformed contact shape, third term pertains to elastic deformation of the contacting surfaces and the last term represents the roughness term with $\bar{a}$ as amplitude and $l$ as wavelength. $H_{0}$ is the integrating constant, i.e., the offset film thickness, determined indirectly by the load balance equation, given in dimensionless form as

$$
\int_{X_{1}}^{X_{c}} P(X) d X=\frac{\pi}{2} .
$$

The dimensionless forms of density $\rho=\rho(P)$ and viscosity $\eta=\eta(P)$, which are functions of pressure, are given by the relations [1].

$$
\rho(P)=1+\frac{0.6 e-9 P p_{h}}{1+1.7 e-9 P p_{h}}
$$

which is valid for both mineral and synthetic lubricants, and

$$
\begin{aligned}
\eta(P)= & \exp \left[\left(\ln \eta_{0}+9.67\right) *\left\{-1+\left(1+5.1 e-9 P p_{h}\right)^{z}\right\}\right] \\
& (\text { Roelands relation }[49])
\end{aligned}
$$

respectively, $z$ is the viscosity index $(\mathrm{z}=0.68), p_{0}$ the ambient pressure $\left(p_{0}=1.96 e+8\right), \alpha$ the pressure viscosity relation $(\alpha=2.165 e-8), p_{h}$ is the maximum Hertizian pressure. The physical dimensionless parameters characterizing the EHL line contact problems are velocity (U), load (W) and elasticity (G) and couple stress $\left(L_{m}\right)$ parameters. The corresponding boundary conditions are

$$
P\left(X_{1}\right)=P\left(X_{c}\right)=0 \quad \text { and } \quad \frac{d P}{d X}=0 \quad \text { at } \quad X=X_{c}
$$

where $X_{c}$ corresponds to cavitation point. 
Table 1. The sensitivity of convergence/non-convergence of numerical scheme to the physical parameters with couple stress parameter and waviness surface $(\mathrm{A}=0.2$ and $l=0.12)$ with grid points $\mathrm{n}=350$ (asymptotic value).

\begin{tabular}{|c|c|c|c|c|c|c|}
\hline \multirow[b]{3}{*}{$\mathrm{U}$} & \multirow[b]{3}{*}{$\mathrm{W}$} & \multirow[b]{3}{*}{$L_{m}$} & \multicolumn{4}{|c|}{ Iterations for convergence } \\
\hline & & & \multicolumn{2}{|c|}{ Newton-Raphson Method } & \multicolumn{2}{|c|}{ JFNMG method } \\
\hline & & & Convergence & CPU time (s) & convergence & CPU time (s) \\
\hline \multirow[t]{5}{*}{$1.0 \mathrm{E}-11$} & $2.0 \mathrm{E}-4$ & 0.0 & Yes (144) & 2.6 & Yes (103) & 2.1 \\
\hline & & $1.0 \mathrm{E}-5$ & Yes (100) & 1.90 & Yes (77) & 1.75 \\
\hline & & $1.0 \mathrm{E}-4$ & Yes (2025) & 27 & Yes (145) & 3.92 \\
\hline & & $1.0 \mathrm{E}-3$ & No & $\ldots$ & Yes (190) & 4.21 \\
\hline & & $1.0 \mathrm{E}-2$ & No & $\ldots$ & Yes (205) & 5.3 \\
\hline \multirow{4}{*}{$1.0 \mathrm{E}-10$} & $2.0 \mathrm{E}-5$ & $1.0 \mathrm{E}-5$ & 36100 & 312.04 & Yes (376) & 9.3 \\
\hline & & $1.0 \mathrm{E}-4$ & 2075 & 18.23 & Yes (147) & 4.02 \\
\hline & & $1.0 \mathrm{E}-3$ & No & $\ldots$ & Yes (412) & 10.92 \\
\hline & & $1.0 \mathrm{E}-2$ & No & $\ldots$ & Yes (472) & 11.73 \\
\hline
\end{tabular}

PRESSURE AND FILM THICKNESS DISTRIBUTION

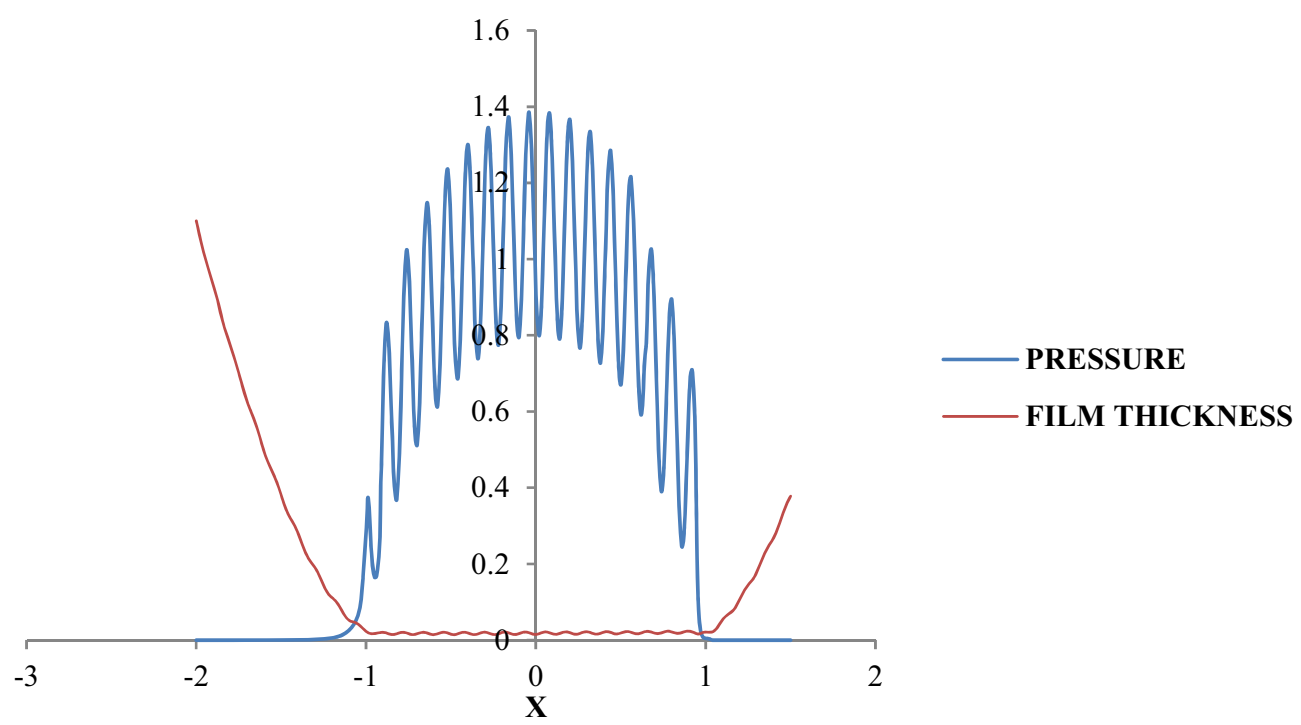

Figure 1. Pressure and film thickness distribution for $\mathrm{W}=2.0 \mathrm{E}-4, \mathrm{U}=1.0 \mathrm{E}-11, \mathrm{G}=5.0 \mathrm{E} 3$ and $L_{m}=0.0$.

Finite difference discretization of the equations The equations (3.1)-(3.3) are discretized using second order finite differences with uniform grid of $n+1$ points (i.e., $n-1$ interior points) $X_{i}, \quad 1 \leq i \leq n+1$, and the domain of interest, initially is $\left[X_{1}, X_{2}\right]=[-2,1.5], X_{c}$, the cavitation point to be determined in the solution process, is an internal point near exit. The discretized form of Reynolds equation is

$$
\begin{aligned}
& \frac{\left(\frac{\varepsilon_{i}+\varepsilon_{i+1}}{2}\right)\left(\frac{P_{i+1}-P_{i}}{\Delta x}\right)-\left(\frac{\varepsilon_{i}+\varepsilon_{i-1}}{2}\right)\left(\frac{P_{i}-P_{i-1}}{\Delta x}\right)}{\Delta x}-K\left(\frac{\rho_{i} H_{i}-\rho_{i-1} H_{i-1}}{\Delta x}\right) \\
& \quad=0
\end{aligned}
$$

where $\varepsilon_{i}=\frac{\rho_{i} H_{i}^{3}}{\eta_{i} \xi_{i}}, \quad \xi_{i}=\left[1-\frac{12}{\Omega_{i}^{2}}+\frac{24}{\Omega_{i}^{3}} \tanh \left(\frac{\Omega_{i}}{2}\right)\right]^{-1}, \Omega_{i}=\frac{8 W H_{i}}{\pi L_{m}}$, $K=\frac{\left(3 U \pi^{2}\right)}{4 W^{2}}$.

The film thickness equation approximated at $X_{i}$ on the regular grid is given by

$$
H\left(X_{i}\right)=H_{0}+\frac{X_{i}^{2}}{2}-\frac{1}{\pi} \sum_{j=1}^{n} K_{i j} P\left(X_{j}\right)+\bar{a} \sin \left(\frac{2 \pi X_{i}}{l}\right)
$$

where

$$
\begin{aligned}
K_{i j}= & \left(X_{i}-X_{j}+\frac{\Delta X}{2}\right)\left(\log \left|X_{i}-X_{j}+\frac{\Delta X}{2}\right|-1\right) \\
& -\left(X_{i}-X_{j}-\frac{\Delta X}{2}\right)\left(\log \left|X_{i}-X_{j}-\frac{\Delta X}{2}\right|-1\right)
\end{aligned}
$$


PRESSURE AND FILM THICKNESS DISTRIBUTION

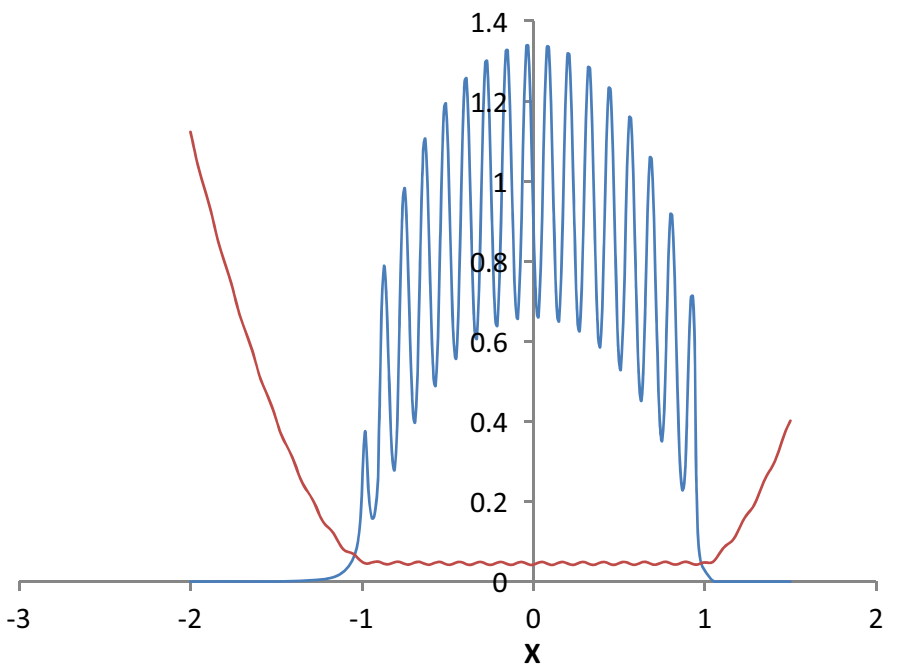

(a)
- PRESSURE

FILM THICKNESS

PRESSURE AND FILM THICKNESS DISTRIBUTION

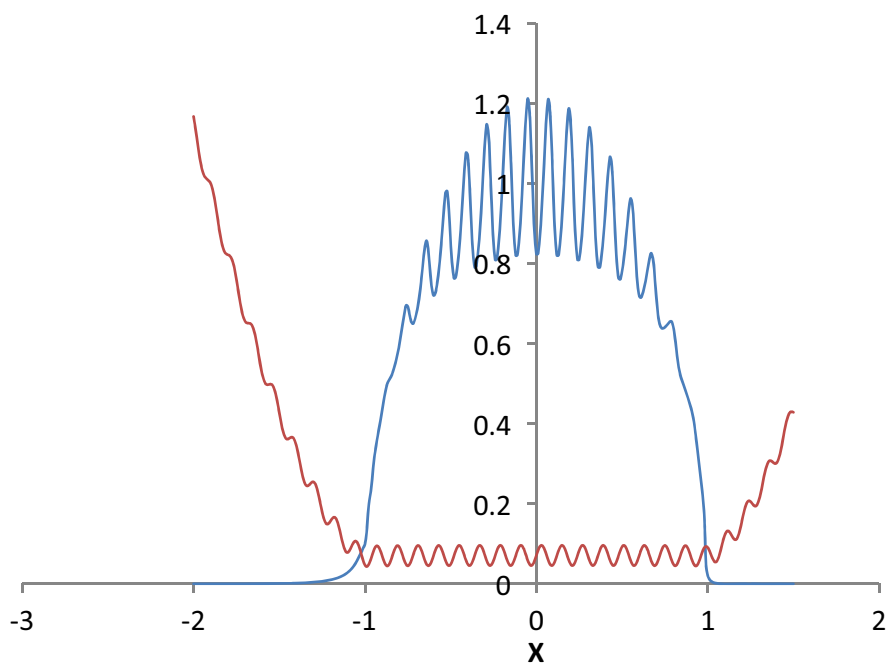

(b)

Figure 2. (a) Pressure and film thickness profiles for $\mathrm{W}=2.0 \mathrm{E}-4, \mathrm{U}=1.0 \mathrm{E}-11, \mathrm{G}=5.0 \mathrm{E} 3$ and $L_{m}=1.0 \mathrm{E}-5$. (b) Pressure and film thickness profiles for $\mathrm{W}=2.0 \mathrm{E}-5, \mathrm{U}=1.0 \mathrm{E}-10, \mathrm{G}=5.0 \mathrm{E} 3$ and $L_{m}=1.0 \mathrm{E}-5$.

For $i=1,2, \ldots, n+1$ and $j=1,2, \ldots, n+1$, and the force balance equation in discrete from is

$$
\Delta X \sum_{j=1}^{n}\left(\frac{P_{j}+P_{j+1}}{2}\right)-\frac{\pi}{2}=0
$$

In discretizing equations (3.2) and (3.3), a piece-wise constant form of $P(X)$ is assumed. The pressure boundary conditions are

$$
P\left(X_{1}\right)=0, \frac{d P}{d X}=0 \text { at } X=X_{c}
$$

The above described EHL line contact problem is investigated using Newton-Raphson technique [36] and convergence of the solution scheme for different combinations of high load-low speed and low load-high speed conditions are presented. The focus of the present paper is to analyze these findings in a more general computational set-up using Jacobian-free Newton multigrid method for obtaining fast converging and accurate solutions for various combinations of parameters of interest and for much larger values of couple stress parameter. 
PRESSURE AND FILM THICKNESS DISTRIBUTION

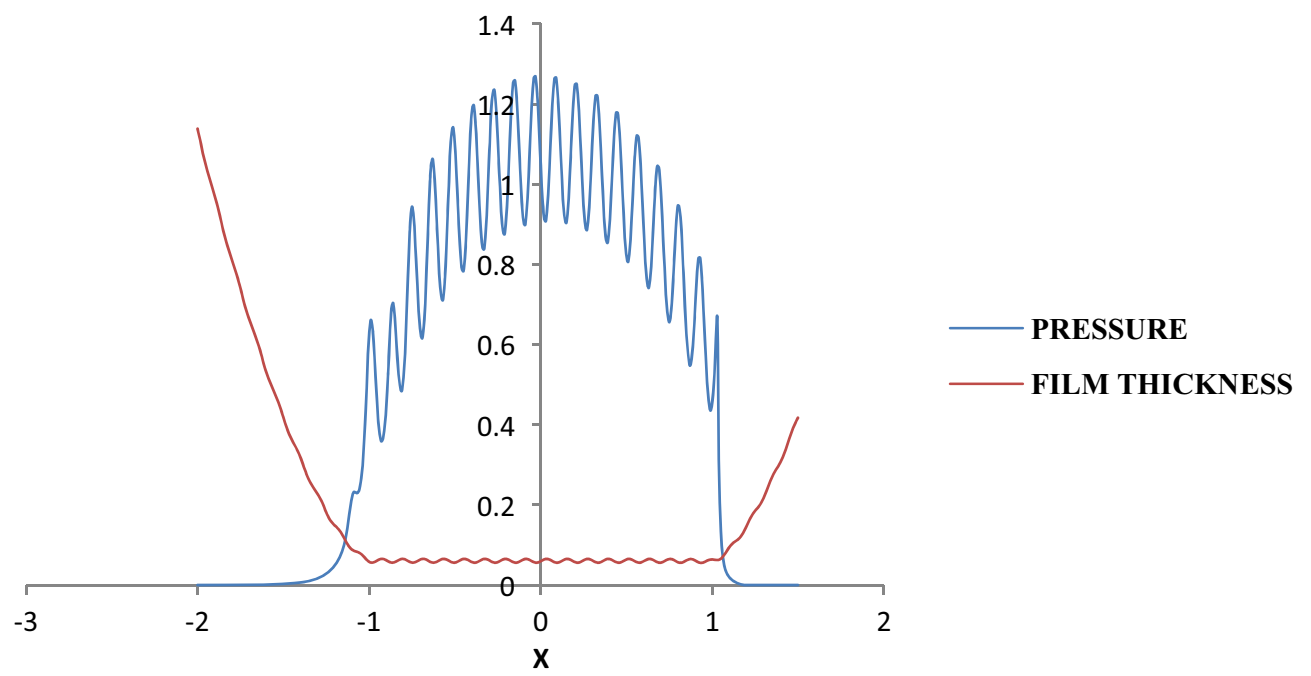

Figure 3. Pressure and film thickness distribution for $\mathrm{W}=2.0 \mathrm{E}-4, \mathrm{U}=1.0 \mathrm{E}-11, \mathrm{G}=5.0 \mathrm{E} 3$ and $L_{m}=1.0 \mathrm{E}-4$.

\section{PRESSURE AND FILM THICKNESS DISTRIBUTION}

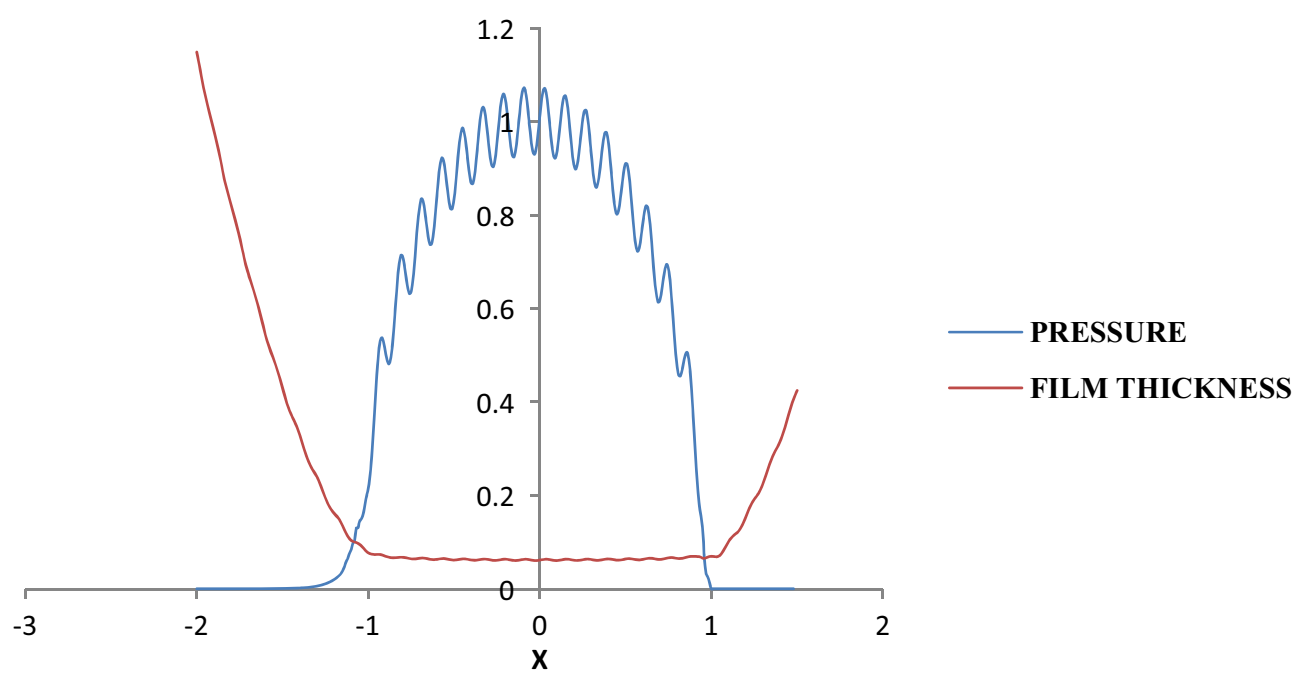

Figure 4. Pressure and film thickness distribution for $\mathrm{W}=2.0 \mathrm{E}-4, \mathrm{U}=1.0 \mathrm{E}-11, \mathrm{G}=5.0 \mathrm{E} 3$ and $L_{m}=1.0 \mathrm{E}-3$.

\section{Numerical implementation}

The discretized systems of equations (4.1)-(4.2) and (4.4) are solved using Jacobian-free Newton Multigrid method. For each Newton iteration (outer loop), there is a linear system to be solved using multigrid method (inner loop). The required Jacobian matrix-vector product is computed using relation (2.6). With this, the pressure-viscosity and pressure-density relations are used in the Jacobian-free computation with much ease. The solution scheme consists of the following steps [50].

Step i Take initial values of $P, X_{c}$ and $H_{0}$
Step ii Evaluate $H$ from film thickness equation (4.2)

Step iii Solve the linear system using multigrid (Vcycles) and use Gauss-Seidel relaxation

Step iv $\quad$ Solve Reynolds equation (4.1) for $P$ and use numerical under-relaxation for stability and convergence of iteration

$P_{n+1}^{\text {new }}=P_{n}^{\text {old }}+C_{1}\left(P_{n}^{\text {new predictor }}-P_{n}^{\text {old }}\right), \mathrm{C}_{1}$ being the under-relaxation parameter

Step v Update $H_{0}$ using force balance equation (4.4) and $H_{0}=H_{0}+R C_{2}$, where $\mathrm{R}$ is residual of the force balance equation, $\mathrm{C}_{2}$ is the underrelaxation parameter 


\section{PRESSURE AND FILM THICKNESS DISTRIBUTION}

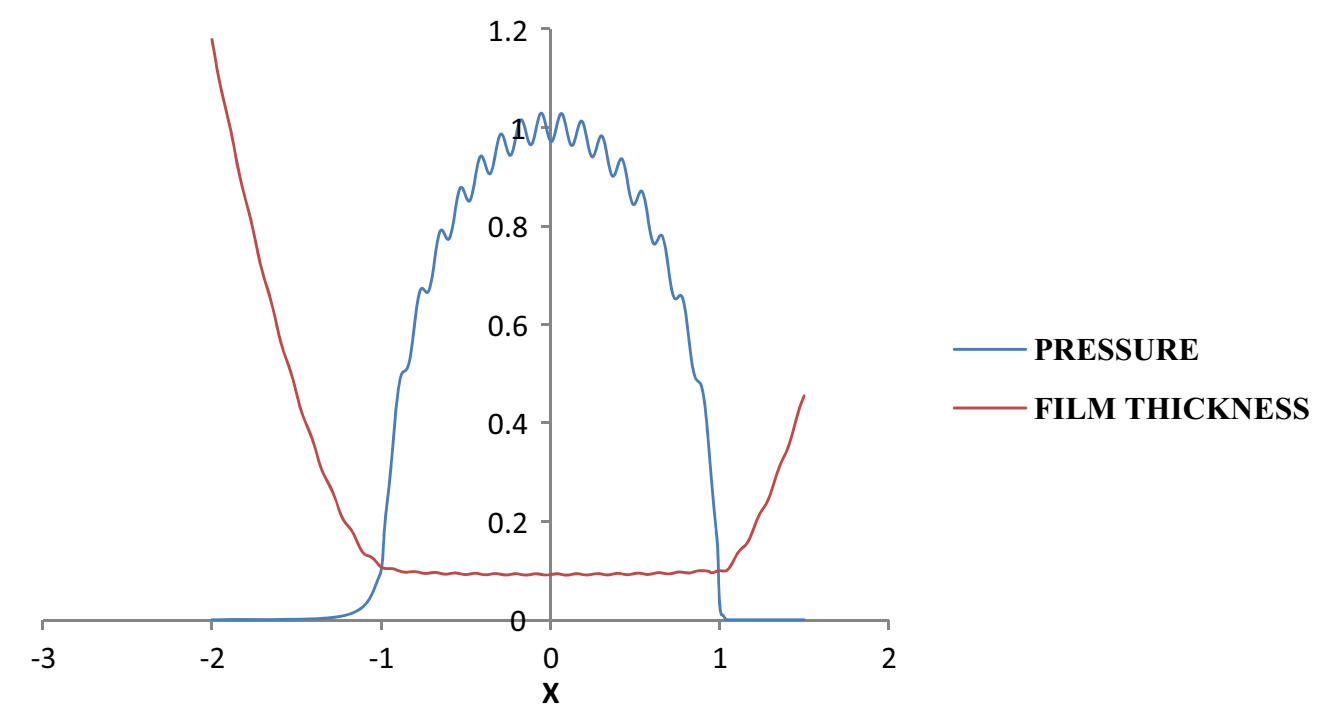

Figure 5. Pressure and film thickness distribution for $\mathrm{W}=2.0 \mathrm{E}-4, \mathrm{U}=1.0 \mathrm{E}-11, \mathrm{G}=5.0 \mathrm{E} 3$ and $L_{m}=1.0 \mathrm{E}-2$.

Step vi Fix the cavitation point $X_{c}$ using $\frac{d P}{d X}=0$ at $X=X_{c}$

Step vii While not converged, go to Step ii

Hertz's pressure distribution is used as initial approximation for pressure P. An elegant procedure [51] enables in finding optimal values of the under-relaxation parameters $C_{1}$ and $C_{2}$. The numerical values of these constants are in the range $0.4<C_{1}<0.8$ and $0<C_{2}<0.1$ for different sets of physical parameters. For the termination of iteration (inner loop), the error tolerance value is $1.0 \mathrm{E}-6$. At the Step iii, for the solution of nonlinear system of equations (4.1) for $P$, we use Gauss-Seidel method. For locating cavitation point, the following criteria is adopted (valid for one-dimensional case). To preserve the regularity of the pressure at the cavitation point $X_{c}, \frac{d P}{d X}=0$. If the pressure gradient is zero at the initial guess $X_{c}$, it is fine. Otherwise, move $X_{c}$ one interval to the left or right depending on whether $\left.\frac{d P}{d X}\right|_{X_{c}}>0$ or $\left.\frac{d P}{d X}\right|_{X_{c}}<0$, respectively. Approximate new cavitation point $X_{c^{\prime}}$ and solve EHL problem. If pressure gradient is zero at $X_{c^{\prime}}$, then we get converged solution. Otherwise, check for the condition whether

$$
\left.\frac{d P}{d X}\right|_{X_{c}} \times\left.\frac{d P}{d X}\right|_{X_{c^{\prime}}}<0
$$

If condition (5.1) is not met, repeat the above process and locate two approximate values $X_{c^{\prime}}$ and $X_{c^{\prime \prime}}$ for which (5.1) is satisfied. Interpolate the cavitation point between the two points $X_{c^{\prime}}$ and $X_{c^{\prime \prime}}$ and call this new point by $X_{I C}$. Relocate the grid points so that $X_{I C}$ is a node point. Solve the system of EHL equations. This ensures cavitation point pressure gradient to be nearly zero at $X_{I C}$ and results into the final required smooth solution of EHL problem. Alternatively, a more general procedure involves representing $\frac{d P}{d X}$ in difference form and clubbing it as an additional equation with difference schemes (4.1), where $X_{c}$ (unknown cavitation point) is to be located suitably. Once accurate solution of linear system is obtained, Newton-inexact scheme [47] (requiring just 4-5 iterations) enables in finding solution for pressure $P$ and height $H$ accurately. These computations are repeated for all sets of physical parameters using Roelands viscosity-pressure relation.

The solution procedure given in the above section is implemented. At the Step iii, Gauss-Seidel relaxation is used to solve the linear system (inner loop) and the convergence criterion adapted at this step is that the multigrid $\mathrm{V}$-cycles are carried until the norm of the residual is less than prescribed tolerance $(1.0 \mathrm{E}-06)$. Using this solution (of linear system) of the inner loop and suitable under-relaxation parameters, the inexact Newton method (Newton line search in outer loop) gives solution of the nonlinear system, which requires 4-5 iterations to yield solution with error norm less than $1.0 \mathrm{E}-06$. The offset film thickness $\mathrm{H}_{0}$ is calculated from the force balance equation using latest pressure values. The cavitation point is then updated using the procedure stated earlier. These computations are repeated, with mesh size $\Delta X=0.01$ for all sets of values of physical parameters of interest. In fact, for smaller values of $L_{m}$, (couple stress parameter) and lower load, much coarser grid is enough to obtain accurate solution. As load and couple stress parameters increase, it requires finer grids for the converging solution. The entire numerical computation is done using MATLAB in double precision. It is of interest to observe that JFNMG performs well compared to Multigrid methods (FMG-FAS) in attaining prescribed 


\section{COMPARISON OF PRESSURE DISTRIBUTION}

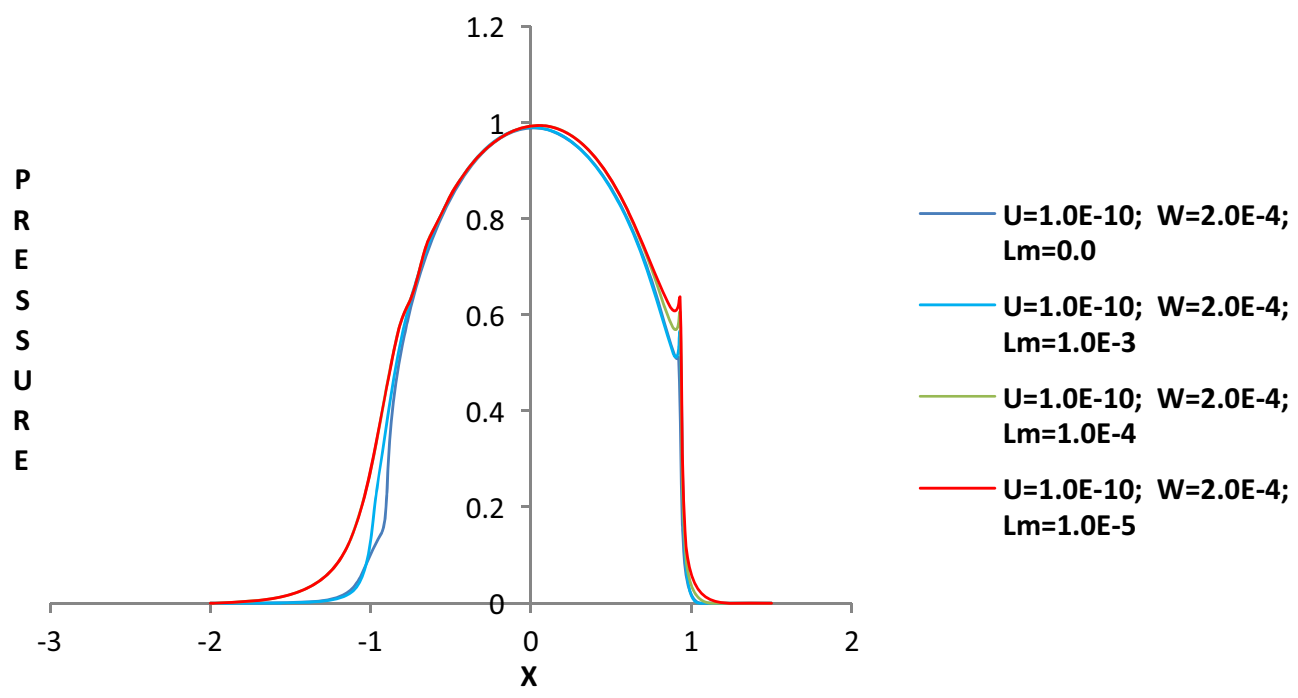

(a)

\section{COMPARISON OF FILM THICKNESS DISTRIBUTION}
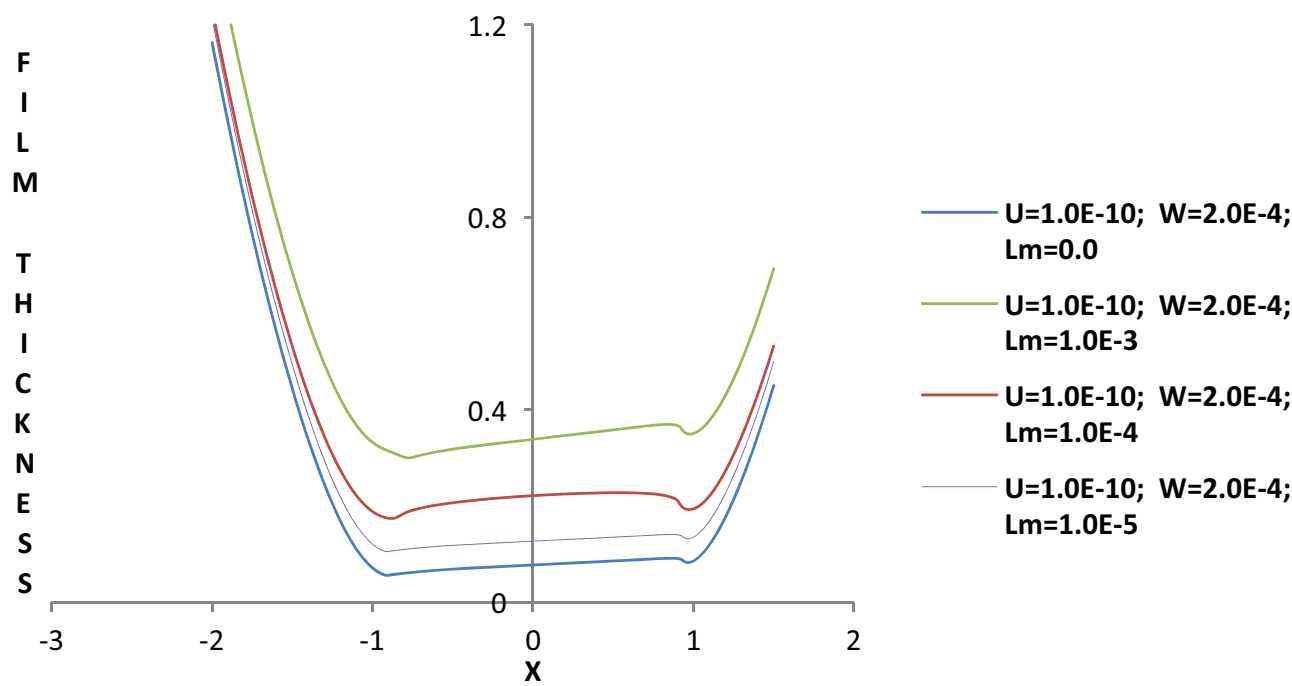

(b)

Figure 6. (a) Pressure profiles for $\mathrm{W}=2.0 \mathrm{E}-4, \mathrm{U}=1.0 \mathrm{E}-10, \mathrm{G}=5.0 \mathrm{E} 3$ and different values of $L_{m}$. (b) Film profiles for $\mathrm{W}=2.0 \mathrm{E}-4, \mathrm{U}=1.0 \mathrm{E}-10, \mathrm{G}=5.0 \mathrm{E} 3$ and different values of $L_{m}$.

accuracy if the inner and outer iterations are suitably selected. Similar predictions are made [16] (page: 105-106) while presenting basic structure of NMG and Multigrid (FMG-FAS) schemes in solving representative nonlinear equations using these schemes. These are the representative nonlinear examples and, in the absence of concrete theory, general claim cannot be made. The mesh size, required for the converging solution, depends on the parameters $\mathrm{U}, \mathrm{W}$ and $\mathrm{G}$. In fact, for heavy load (and also for high speed), it requires finer grids to solve the system up to desired accuracy.

\section{Results and discussions}

In section 1.1, the importance of couple stress fluid as lubricant is presented. The governing isothermal steady EHL line contact equations are solved using Finite difference scheme with uniform grid. The detail analysis is both for smooth surface as well as rough surface (waviness). For the analysis of rough surface, influence of thermal and transient aspects are important, but here the focus is on demonstrating the robustness of the proposed JFNMG method in obtaining accurate solution of the nonlinear 


\section{COMPARISON OF PRESSURE DISTRIBUTION}

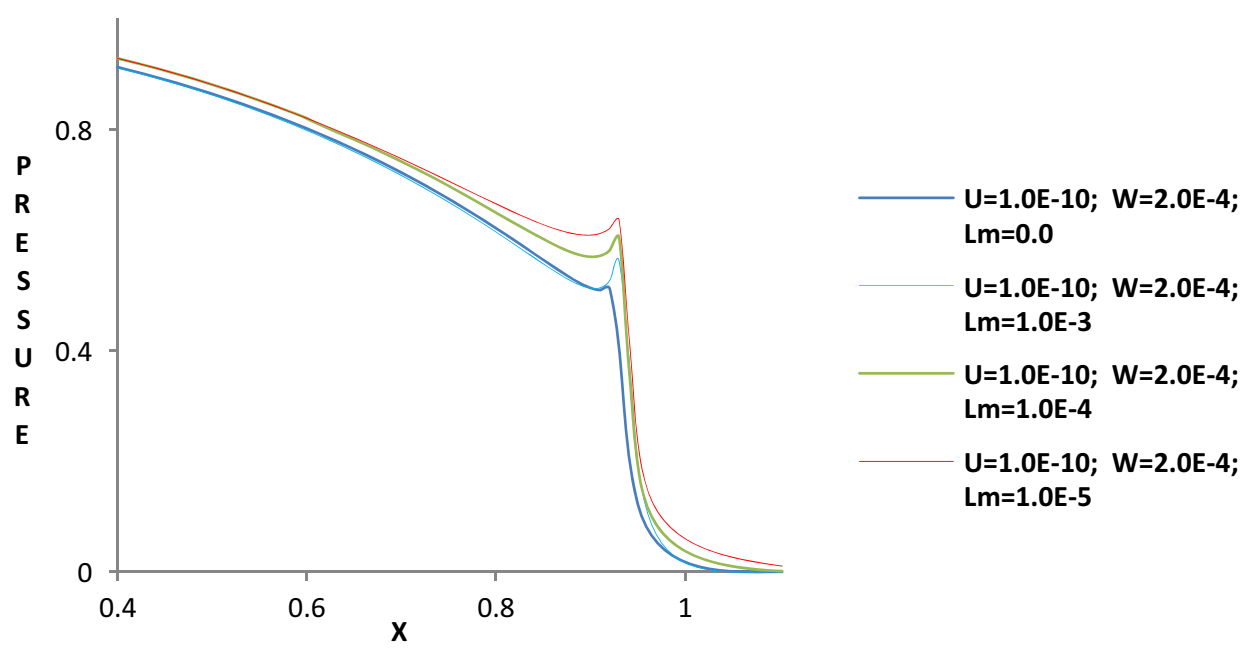

(a)

COMPARISON OF FILM THICKNESS
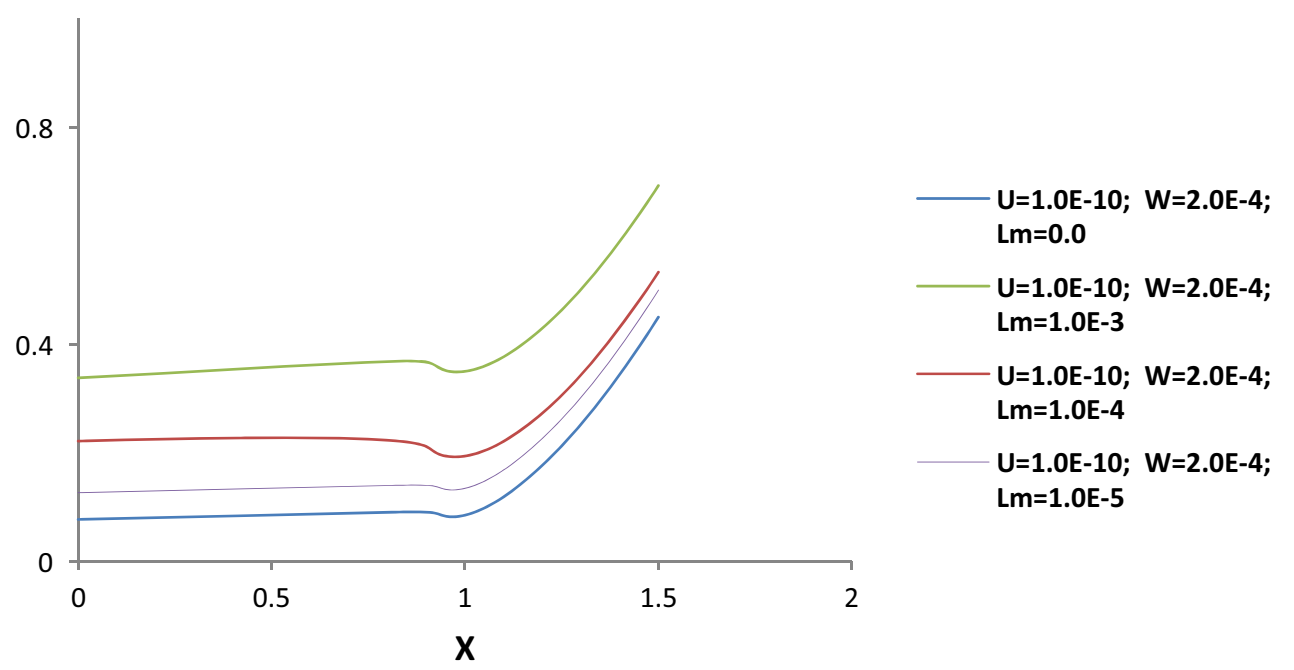

(b)

Figure 7. (a) Spread of pressure profiles for $\mathrm{W}=2.0 \mathrm{E}-4, \mathrm{U}=1.0 \mathrm{E}-10, \mathrm{G}=5.0 \mathrm{E} 3$ and different values of $L_{m}$. (b) Spread of film profiles for $\mathrm{W}=2.0 \mathrm{E}-4, \mathrm{U}=1.0 \mathrm{E}-10, \mathrm{G}=5.0 \mathrm{E} 3$ and different values of $L_{m}$.

system of algebraic equations resulting from the use of finite difference scheme for wide range of parameters of interest.

Table 1 contains the comparison of the sensitivity of conventional NR method with that of JFNMG scheme for various values of $\mathrm{U}, \mathrm{W}$ and $L_{m}$. The NR method does not converge even for moderately low values of $\mathrm{U}, \mathrm{W}$ or $L_{m}$ whereas JFNMG is fast converging and works for wide range of EHL parameters of interest. Figure 1 shows the pressure and film profiles for Newtonian case $\left(L_{m}=0\right)$ with $\mathrm{A}=0.2, l=0.12, \mathrm{U}=1.0 \mathrm{E}-11$ and $\mathrm{W}=2.0 \mathrm{E}-4$. The nonNewtonian results depicted in figures 2(a), 2(b), 3, 4 and 5 (for different values of $L_{m}$ ) show increase in film thickness and suppression of the wriggles in the profiles due to surface roughness in comparison with the Newtonian results (figure 1).

Smooth surface with couple stress fluid as lubricant: The solution obtained using JFNMG, for the pressure and film shape are for various combinations of parameters, namely, $\mathrm{U}, \mathrm{W}, \mathrm{G}, L_{m}$ (couple stress), $\bar{a}$ (amplitude) and $l$ (wavelength) for smooth as well as wavy rough surface. Earlier investigators [36] on this problem used Newton-Raphson method and presented useful findings. The method converges only for moderate values of couple stress parameter $L_{m}$, whereas in the present analysis it is possible to obtain stable and converging solution of the problem for larger 
values of $L_{m}$ as well as for various other combinations of parameters involved. In figure 6(a), pressure profiles are shown for smooth surfaces, for one set of U, W, G and for various values of $L_{m}$ while in figure 6(b), profiles of associated film thickness are given. The pressure spike (spread as well as its height) almost shrinks (diminishes) for increasing values of $L_{m}$. These are associated with increase in minimum film thickness and corresponding dip at the spike (location) of the film profile. In figures 7(a) and (b), the spread forms of pressure spike and film shapes, respectively are shown for various values of $L_{m}$. These findings validate experimental observations, on lubricants with additives, by earlier studies.

\subsection{Rough surface with couple stress fluid as lubricant}

For the wavy rough surface, the pressure and film shapes are functions of amplitude $\bar{a}$ and wavelength $l$, in addition to their sensitivity to $L_{m}, \mathrm{U}, \mathrm{W}$ and G. Figures 2(a) and (b) pertain to pressure and film profiles for high load-low speed and low load-high speed, respectively, for $\bar{a}=0.2, l$ $=0.12$ and $L_{m}=1.0 \mathrm{E}-5$. For the given values of $\bar{a}=0.2$, $l=0.12$, and one set of $\mathrm{U}, \mathrm{W}, \mathrm{G}$, the pressure and film profiles are shown in figures 3, 4 and 5 for increasing values of $L_{m}(1.0 \mathrm{E}-4$ to $1.0 \mathrm{E}-2)$. We find considerable decrease in wriggles of the profiles compared to those corresponding to figures for $L_{m}=1.0 \mathrm{E}-5$. These findings confirm that the lubricant with additives suppress the wriggles in film profiles corresponding to larger values of the couple stress parameter $L_{m}$. These predictions enable designers in arresting bearing fatigue life.

\section{Conclusion}

An important issue of computing the converging solution of EHL line contact problem using JFNMG method is addressed here. It is observed that, for the investigation of the influence of pressure-viscosity and pressure-density nonlinear relations on EHL characteristics, Jacobian-free numerical scheme is the most effective one. It is possible to cover wide range of load conditions (of physical parameters) using Roelands pressure-viscosity relations. The proposed JFNMG scheme enables one to compute Jacobian matrix-vector product, at each iteration, of the inner loop (linear multigrid) accurately with much ease without requiring any additional storage for the Jacobian. It also overcomes other drawbacks of Newton-Raphson method, namely, the difficulty of implementing cavitation condition, and avoids huge requirement of computer memory and CPU time (whenever grid size is halved in order to minimize discretization error). Also, implementation strategy such as finding optimal relaxation parameters leading to the convergence of the scheme is given. Besides obtaining accurate pressure distribution and pressure-height relation, the nature of pressure spike as a function of load and couple stress parameter is demonstrated. The proposed JFNMG method inherits convergence features of Newton method as well as convergence strategy (up to discretization error) of Multigrid. This work motivates for the future study in applying the proposed scheme (or its variants) to more involved EHL problems, namely, for the analysis of thermal and non-Newtonian rheology of lubricant on EHL characteristics. Further, it can be extended to the investigation of point contact problem, starvation and other current topics of interest in EHL.

\section{Acknowledgements}

Authors thank the Department of Science and Technology (SR/S4/MS: 771/12) and Indian National Science Academy (SP/HIS/2012/425), New Delhi, India for the financial support. Also, thank the reviewers for their useful suggestions and comments on the earlier draft of the manuscript.

\section{List of symbols}

a Amplitude of roughness $(\mu \mathrm{m})$

$\bar{a} \quad$ Dimensionless amplitude of roughness, $\bar{a}=a R / b^{2}$

A Normalized surface roughness amplitude, $A=\bar{a} / H_{\mathrm{min}}^{D H}$

$b \quad$ Half width of the Hertzian contact, $b=4 R \sqrt{W / 2 \pi}$

$E^{\prime} \quad$ Effective elastic modulus of rollers 1 and $2(\mathrm{~Pa})$

$G \quad$ Dimensionless materials parameter, $\alpha E^{\prime}$

$h \quad$ Film thickness (m)

$H \quad$ Dimensionless film thickness, $H=h R / b^{2}$

$h_{0} \quad$ Offset film thickness (m)

$H_{0} \quad$ Dimensionless constant/offset film thickness $H_{0}=h_{0} R / b^{2}$

$K_{i j} \quad$ Discrete approximation of $K$ logarithmic kernel Eq. (4.3)

$l \quad$ Dimensionless wavelength of roughness, $l=\lambda / b$

$L_{m} \quad$ Dimensionless couple stress parameter, $L_{m}=\lambda_{a} / R$

$N \quad$ Number of nodes on grid

$p \quad$ Pressure (pa)

$P_{h} \quad$ Maximum Hertzian press $P_{h}=(2 w) /(\pi b)$

$P \quad$ Dimensionless pressure, $P=p / P_{h}$

$P_{0} \quad$ Ambient pressure

$R \quad$ Equivalent radius of contact

$u \quad$ Velocity component

$u_{1}, u_{2} \quad$ Velocities of lower and upper surfaces respectively $(\mathrm{m} / \mathrm{s})$

$u_{s} \quad$ Sum velocity, $u_{s}=\left(u_{1}+u_{2}\right) / 2$

$U \quad$ Dimensionless speed parameter, $U=\left(\eta_{0} u_{s}\right) /\left(E^{\prime} R\right)$ 
$v \quad$ Surface displacement (m)

$\bar{v} \quad$ Dimensionless displacement, $\bar{v}=v R / b^{2}$

$w \quad$ External load per unit width

$W \quad$ Dimensionless load parameter, $W=w /\left(E^{\prime} R\right)$

$\Delta X \quad$ Mesh size

$x \quad$ Abscissa coordinate

$X \quad$ Dimensionless coordinate $x / b$

$\left[X_{1}, X_{2}\right] \quad$ Domain of interest $[-2,1.5]$

$X_{c} \quad$ Dimensionless location of pressure spike

$z \quad$ Pressure viscosity parameter

$\alpha \quad$ Pressure viscosity index

$\eta \quad$ Fluid viscosity

$\eta_{0} \quad$ Viscosity at ambient pressure

$\bar{\eta} \quad$ Dimensionless viscosity, $\eta / \eta_{0}$

$\lambda \quad$ Surface roughness wavelength $(\mu \mathrm{m})$

$\lambda_{a} \quad$ Molecular length of additives (m)

$\xi \quad$ Viscosity modification factor

$\rho \quad$ Lubricant density at local pressure

$\rho_{0} \quad$ Inlet density of the lubricant

$\bar{\rho} \quad$ Dimensionless fluid density, $\rho / \rho_{0}$

\section{References}

[1] Dowson D and Higginson G R 1977 Elasto-hydrodynamic lubrication, Second Edition. Oxford: Pergamon Press, pp 1-19

[2] Hamrock B J and Dowson D 1976 Isothermal lastohydrodynamic lubrication of point contacts part I, J. Lub. Tech. Trans. ASME 98: 223-229

[3] Hamrock B J and Dowson D 1981 Ball bearing lubrication. New York: Wiley

[4] Hamrock B J and Jacobson B O 1984 Elastohydrodynamic lubrication of line contacts. ASLE Trans. 27(4): 275-287

[5] Chittenden R J, Dowson D, Dunn J F and Taylor C M 1985 A theoretical analysis of the isothermal elastohydrodynamic lubrication of concentrated contacts I: direction of lubrication entrainment with the major axis of the horizontal contact elliptic. Proc. R. Soc. Lond. A 397: 245-269

[6] Chittenden R J, Dowson D, Dunn J F and Taylor C M 1985 A theoretical analysis of the isothermal elastohydrodynamic lubrication of concentrated contacts II: general case with lubricant entrainment along either principal axis of the Hertizan contact ellipse or at some intermediate angle. Proc. R. Soc. Lond. A 397: 271-294

[7] Okamura H 1982 Contribution to the numerical analysis of elastohydrodynamic lubrication. In: Proceedings of the Leeds-Lyon Symposium on Tribiology, pp 313-320

[8] Petrusevich A I 1951 Fundamental conclusions from the contact hydrodynamic theory of lubrication. Izv. Akad, Nauk SSR (OTN) 3: 209-223

[9] Kostreva M M 1984 Pressure spike and stability considerations in elastohydrodynamic lubrication models. Trans. ASME J. Tribol. 106: 386-395

[10] Kostreva M M 1984 Elasto-hydrodynamic lubrication: a nonlinear complementary problems. Int. J. Num. Methods Fluid 4: 377-397
[11] Habachi W and Issa J 2013, Fast and reduced full system finite element solution of elastohydrodynamic lubrication problem: line contact. Adv. Eng. Softw. 56: 5-62

[12] Lubrecht A A 1987 Numerical solution of EHL line and point contact problems using multigrid techniques. Ph.D., Thesis, University of Twente, Enschede, The Netherlands

[13] Brandt, A and Lubrecht, A A 1990 Multi-level matrix multiplication and fast solutions of integral equations. J. Comput. Phys. 90(2): 348-370

[14] Venner, C H and Lubrecht A A 2000 Multilevel methods in lubrication. London: Elsevier, pp 57-100

[15] Nurgat E, Berzins and Scales L E 1999 Solving EHL problems using iterative methods and homotopy methods. Trans. ASME J. Tribol. 12(1): 28-34

[16] Briggs W L, Henson V E and McCormick S F 2000 A multigrid tutorial, 2nd Edition. Philadelphia: SIAM, pp 105-106

[17] Houpert L G, Ioannides E, Kuypers J C and Tripp J 1987 The effect of the EHD pressure spike on rolling bearing fatigue. ASME J. Tribol. 109: 444-450

[18] Hamrock, B J, Pan P and Lee R T 1988 Pressure spike in EHL lubricated conjunctions. ASME J. Tribol. 110: 279-284

[19] Bissett E J and Glander D W 1988 A highly accurate approach that resolves the pressure spike of EHL. Trans. ASME J. Tribol. 110: 241-246

[20] Venner C H and Napel W E T 1989 Numerical calculations of the pressure spike in EHL. Lub. Sci. 2: 321-334

[21] Schlijper A G, ScalesL E and Rycroft J E 1996 Current tools and techniques in EHL modeling, Tribol. Int. 29: 669-673

[22] Lugt P M and Morales-Espejel G E 2011 A review of elastohydrodynamic lubrication theory. Tribol. Trans. 54: 470-496

[23] Bujurke N M, Kantli M H and Bharati M Shettar 2017 Wavelet preconditioned Newton-Krylov method for elastohydrodynamic lubrication of line contact problems. Appl. Math. Model. 46: 285-298

[24] Kantli M H, Bharati M Shettar and Bujurke N M 2017 Jacobian-free Newton-GMRES method for analysing combined effects of surface roughness and couple stress character of lubricant on EHL line contact. Proc. Indian Nat. Sci. Acad. 83(1): 175-196

[25] Oliver D R and Shahidullah M 1983 Load enhancement effects by polymer-thickened oils in short squeeze film flow. J. Non-Newton Fluid Mech. 3: 93-102

[26] Oliver D R 1988 Load enhancement effects due to polymer thickening in a short model journal bearings, J. non-Newtonian Fluid Mech. 30: 185-196

[27] Spikes H A 1994 The behavior of lubricants current understanding and future possibilities. Proc. Inst. Mech. Eng. 28: 3-15

[28] Scott W and Sunniwattann P 1995 Effects of additives on performance of wet friction clutch materials. Wear 183: $850-855$

[29] Guangteng G, Smeeth M, Cann P M and Spikes H A 1996 Measurement and modeling of boundary film properties of polymeric lubricant additives. In: Proceedings of the Institution of Mechanical Engineers, Part J. The Journal of Engineering Tribology, pp 210-215

[30] Guangteng G and Spikes H A 1996 Fractionation of liquid lubricants at solid surfaces. Wear 200: 336-345 
[31] Stokes V K 1966 Couple tresses in fluids. Phys. Fluids 9: 1709-1715

[32] Chippa S P and Sarangi M 2013 Elastohydrodynamically lubricated finite line contact with couple stress fluids Tribol. Int. 67: 11-20

[33] Das N C 1997 Elastohydrodynamic lubrication theory of line contacts couple stress fluid model, Tribol. Trans. 40: 353-359

[34] Sarangi M, Majumdar B C and Sarkar A 2009 Elasthydrodynamically lubricated ball bearings in couple stress fluid, Part-I. Steady state analysis. Tribol. Trans. 48: 404-414

[35] Saini P K, Kumar P and Tandon P 2007 Thermal elasthydrodynamic lubrication characteristics of couple stress fluids in rolling/sliding line contact. Proc. Inst. Mech. Eng. Part J, J. Eng. Tribol. 221: 141-153

[36] Saini P K, Kumar P and Tandon P 2008 Surface roughness effects in elastohydrodynamic lubrication line contact using couple stress fluid. Proc. Inst. Mech. Eng. Part J 222: 151-15

[37] Chu L M, Li W L, Lin J R and Chang R P 2010 Coupled effects of surface roughness and flow rheology on elastohydrodynamic lubrication. Tribol. Int. 43: 483-490

[38] Patir N and Cheng, H S 1978 An average flow model for determine effects of three Ddmensional roughness on partial hydrodynamic lubrication. ASME J. Lub. Technol. 100: $12-17$

[39] Christensen H 1970 Stochastic models for hydrodynamic lubrication of rough surfaces. Proc. Inst. Mech. Eng. 1969-70 184 (Part 1, 55): 1013-1026

[40] Shi F and Wang Q 1998A mixed-TEHD model for journal bearing conformal contacts, part I: models formulation and approximation of heat transfer considering asperity contacts. ASME J. Tribol. 120: 198-205

[41] Tripp J H and Hamrock B J 1985 Surface roughness effects in elastohydrodynamic contacts. In: Proceedings of the 1984 Leeds-Lyon Symposium on Tribology, pp 30-39
[42] Kumar P and Kumar N 2014 Surface roughness effects in ure sliding EHL line contact with carreau-type sheer thinning lubricant. Int. J. Mech. Aerosp. Industrial Mech. Eng. 8(6): 1087-1092. World Academy of Sciences, Engineering Technology

[43] Ioannides E and Harris T A 1985 A new fatigue life made for rolling bearing. ASME J. Tribol. 107: 367-378

[44] Xu G and Sadeghi F 1996 Thermal EHL analysis of circular contacts with measured surface roughness. ASME J. Tribol. 118: 473-482

[45] Zhu D and Hu YZ 2001 A computer program package for the prediction of EHL and mixed lubrication characteristics, friction, subsurface stresses and flash temperatures based on measured 3-D surface roughness. Tribol. Trans. 44: 383-390

[46] Wang W Z, Liu Y C, Hu Y Z, Wang H and Zhu D 2003 A comparative study of the methods for calculation of surface elastic deformation. Proc. Inst. Mech. Eng. Contacts Part J, J. Eng. Tribol. 217(J2): 145-153

[47] Kelley C T 1999 Iterative methods for solving linear and nonlinear equations Philadelphia: Society for Industrial and Applied Mathematics (SIAM), pp 135-144

[48] Knoll D A and Keyes D E 2004 Jacobian free NewtonKrylov methods: a survey of approaches and applications. $J$. Comput. Phys. 193: 357-397

[49] Roelands C J A 1996 Correlational aspects of the viscositytemperature-pressure relationship of lubricating oils. $\mathrm{PhD}$ Thesis, Technische Hogeschool Delft, V.R.B., Groningen, The Netherlands

[50] Zargari E A, JimackP K and Walkley M A 2007 An investigation of the film thickness calculation for elastohydrodynamic lubrication problems. Int. J. Numer. Math. Fluids 00: $1-6$

[51] Durbin T and Delemos D 2007 Adaptive under-relaxation of picard iterations in ground water models. Ground Water 45(5): 648-651 